\title{
Potential and status of damage controlled forming processes
}

\author{
Gerhard Hirt $^{1}$ - A. Erman Tekkaya ${ }^{2} \cdot$ Till Clausmeyer $^{2} \cdot$ Johannes Lohmar $^{1} \mathbb{C}$
}

Received: 19 December 2019 / Accepted: 19 December 2019 / Published online: 14 January 2020

(c) The Author(s) 2020

\begin{abstract}
In modern process design of metallic components, the influence of the metal forming process on the component properties can be taken into account. However, damage occurring concurrent to forming cannot be accounted for yet. Due to the complex multi-scale, multi-mechanism nature of damage, it is very challenging to predict its evolution through any metal forming process chain. In order to enable such damage controlled forming processes in the future three research questions need to be addressed in detail: How are the mechanisms governing the damage initiation and evolution in metals best characterized? How can the damage mechanisms be described and the damage evolution be predicted using models? How do forming processes influence the damage evolution? Answering these questions and considering damage during process design will in the long term lead to improved lightweight components that do not require conventional safety factors, as their performance is already fully known.
\end{abstract}

Keywords Metal forming $\cdot$ Damage $\cdot$ Usability $\cdot$ Forming process chains

\section{Introduction and motivation}

One of the essential engineering challenges lies in the supply of highly durable, efficient components for applications such as transportation and structural engineering. Lightweight components will continue to play a vital role in meeting the requirements of sustainability and resource efficiency, while at the same time complying with the highest demands in terms of the reliability of these components and systems in the foreseeable future [1].

Forming processes are of utmost importance for the production of lightweight components made of metallic materials. This outstanding position on the one hand is due to the high productivity and robustness of the processes as well as the excellent and reliable quality level of the formed parts. On the other hand, the efficient material utilization in forming processes results in low embodied energy in the

Gerhard Hirt

gerhard.hirt@ibf.rwth-aachen.de

https://www.ibf.rwth-aachen.d

1 Institute of Metal Forming, RWTH Aachen University, Intzestraße 10, 52072 Aachen, Germany

2 Institute of Forming Technology and Lightweight Components, TU Dortmund University, Baroper Str. 303, 44227 Dortmund, Germany product [2]. Additionally, forming processes not only set the geometric shape and surface, but also the mechanical properties of the metallic component. A positive influence on the mechanical properties results in particular from work hardening and microstructure evolution e.g. via phase transformations or grain refinement due to recrystallization. At the same time, a certain degree of ductile material damage inevitably occurs during forming and mostly has a negative effect on the mechanical properties.

Despite of that damage has not yet been taken into account in the design of products manufactured by metal forming, as these currently only have to be "failure-free", but not "damage-free". A plentitude of definitions for damage were published in the past either focusing on the difference between relating it to a specific area or volume [3] or on the determination in measurement [4]. Some of these are contradictive, especially when they stem from different disciplines. In the context of damage controlled forming processes the authors suggest the following definition: "Ductile damage causes the degradation of physical properties, in particular of mechanical properties, due to microstructural defects. These defects are characterized by the formation of new surfaces. In structural materials voids and micro cracks initiate mostly by decohesion at interfaces such as grain boundaries and inclusions or due to brittle fracture of hard phases. The evolution of voids and micro cracks then causes damage 
under thermo-mechanical loading." The involved scales, the underlying mechanisms and the course of ductile damage are shown in Fig. 1.

This complex multi-scalar, multi-mechanism nature of damage makes it difficult to consider in manufacturing. However, if the damage level in a metal formed component is not known, there are two direct consequences:

- When designing formed components, light weighting potential is not fully exploited because damage cannot be taken into account and higher safety factors have to be used instead.

- When designing the forming process itself, not all influencing factors on the performance of the component are taken into account.

An increased awareness is particularly relevant for modern high-performance steels. Due to their complex microstructure, they exhibit ductile damage mechanisms, some of which are not fully understood. Void nucleation in single phase metals occurs by decohesion of inclusions from the surrounding matrix or by inclusion cracking [5]. The second mechanism is the growth of voids, which is related to large values of triaxiality, i.e. the hydrostatic stress divided by the equivalent stress [6]. These stages are then followed by void coalescence, which describes the linkage of separate voids as observable today in computer-tomography [7]. In dual or multi-phase materials such as dual phase steels, these mechanisms compete [8] and occur already at very small plastic deformations.

Thus, to exploit fully the potential of such modern material concepts, a paradigm shift in dealing with damage towards damage-controlled forming processes is imperative.

\section{Vision and scope}

The aim of damage-controlled forming processes is to understand and predict material damage during forming and to amend it specifically with regard to component performance. For this purpose, methods for the model-based description of the damage evolution taking into account the governing metal-physical mechanisms as well as technologies for the detection of damage and technical utilization require continuous development.

The basis and starting point is the observation that different process routes for forming a component from a given semi-finished product lead to the same final geometries, but different properties and damage. Thus, it is in principle possible to influence the damage evolution throughout the entire forming process chain-from the semi-finished product to the finished component-via the selection and design of the forming processes and their parameters. If not only the nominal material properties are used in component design, but the damage caused by the forming process is taken into account in the same way as other production-induced properties (cf. Fig. 2), this leads to the paradigm shift mentioned above that effects both the design of the forming processes and the finished components.

The production process design based on maximum formability, which is oriented only towards the failure-free manufacturing of the component, is then superseded by a damage-based process design, which instead considers the usability of the component in terms of the achievement of maximum possible performance. The damage-controlled design of the forming process chain ensures that not only
Fig. 1 The process from damage initiation, via damage evolution to component failure spans different length scales

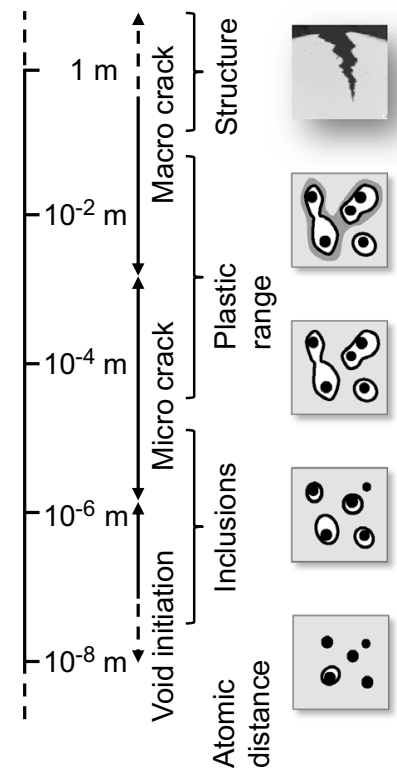

Failure

Discontinuity/decohesion with a size of $\sim 1 \mathrm{~mm}$ after passing the critical damage

Critical damage

Level of accumulated damage leading to material instability or failure

\section{Material decohesion}

Local material decohesion due to accumulated damage

Accumulated damage Initiation, evolution and interaction of microstructural defects due to mechanical or thermal load

\section{Damage}

Local material discontinuity in the form of microstructural defects 
Fig. 2 Principle idea of the damage controlled component design

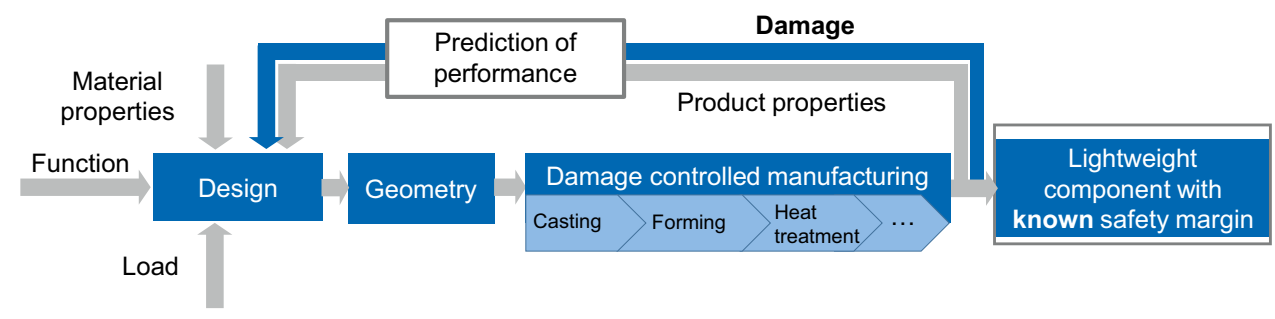

the desired microstructure and the macroscopic properties are set as required, but also that the accumulated damage in the product is minimized. As a result, the component is not only macroscopically failure-free and meets the conventional quality requirements, but also holds superior mechanical properties and a higher load carrying capability.

In order to enable the widespread consideration of damage in metal forming process chains, the following fundamental questions must be addressed that have so far only been touched upon to a limited extent in current research:

- Research of damage-controlled forming processes In order to be able to specifically adjust the damage during forming, it is necessary to understand how the load paths (strain path, temperature, triaxiality, load angle, etc.) can be influenced in the individual forming processes and how these load paths interact with the metal-physical damage mechanisms. An important aspect is to survey methods of controlling the damage level in classical forming processes and hence increase the performance of the components. First applications of this approach for sheet forming and bulk forming are presented in [9].

- Research of measuring techniques for the quantitative detection of damage In order to develop a model description of damage, the metal-physical damage mechanisms located on different length scales must be precisely resolved. In order to describe the damage initiation phase in multi-phase steels in particular, high-resolution characterization methods, such as in-situ testing technology in scanning electron microscopy, must be developed further. While ductile damage is usually the dominant mechanism during forming, the performance of a component in many cases also depends on its cyclic strength. Here it is to be expected that pores caused by ductile damage influence in particular the incubation and short crack growth phases during subsequent cyclic loading. For this reason, the fundamental question must be answered which experimental methods can characterize, quantify and separate the interaction of ductile and cyclic damage mechanisms. A recent comparison of several methods to determine the damage value [4] concludes that different methods can yield quantitatively different results where some additionally exhibit large margins of uncertainty.
- Modelling and prediction of the initiation and development of damage Although the modelling of damage is not fundamentally new and a large number of different, partly empirical models are known in literature, their validation is usually only based on a specific application, so that the knowledge gained cannot be generalized. Accordingly, the fundamental questions as to which different mechanisms govern the different forming processes and how these mechanisms are influenced by common process parameters are still largely unknown. Similarly, the possibilities for healing damage and the effect of damage on component performance are not yet fully understood. These deficits are due to the intrinsic multi-scale nature of the damage: Damage on the macroscopic scale (e.g. a crack) depends on damage on the microscale (e.g. initiation and development of microcracks and micropores). Furthermore, in contrast to other multi-scale problems in modeling, damage is usually associated with material softening. This firstly results in the necessity of regularization as highlighted before [3] and secondly in the fact that damage can merely be described by classical homogenization methods so that further research is required.

The comprehensive consideration of damage in metal forming process chains is pursued within the ongoing collaborative research center TRR188 on "Damage Controlled Forming Processes" by researchers of different disciplines at the TU Dortmund University, the RWTH Aachen University, the Max-Planck-Institut für Eisenforschung and the Brandenburg University of Technology. At least one of the aforementioned research questions is at the center of each of the following articles in this special issue-based on contributions from the TRR188 and from researchers around the world. This issue as a whole thus provides an insight into the current state of research on damage controlled forming processes.

Acknowledgements The authors gratefully acknowledge the funding by the Deutsche Forschungsgemeinschaft (DFG, German Research Foundation) within the TRR 188-Project number 278868966 on "Damage Controlled Forming Processes". Open Access funding provided by Projekt DEAL. 
Open Access This article is licensed under a Creative Commons Attribution 4.0 International License, which permits use, sharing, adaptation, distribution and reproduction in any medium or format, as long as you give appropriate credit to the original author(s) and the source, provide a link to the Creative Commons licence, and indicate if changes were made. The images or other third party material in this article are included in the article's Creative Commons licence, unless indicated otherwise in a credit line to the material. If material is not included in the article's Creative Commons licence and your intended use is not permitted by statutory regulation or exceeds the permitted use, you will need to obtain permission directly from the copyright holder. To view a copy of this licence, visit http://creativecommons.org/licenses/by/4.0/.

\section{References}

1. Allwood J, Cullen J (2011) Sustainable materials—with both eyes open: future buildings, vehicles, products and equipment-made efficiently and made with less new material (without the hot air). UIT Cambridge Ltd, Cambridge

2. Tekkaya AE, Allwood JM, Bariani PF, Bruschi S, Cao J, Gramlich S, Groche P, Hirt G, Ishikawa T, Löbbe C, Lueg-Althoff J, Merklein M, Misiolek WZ, Pietrzyk M, Shivpuri R, Yanagimoto J (2015) Metal forming beyond shaping: Predicting and setting product properties. CIRP Ann Manuf Technol 64(2):629-653
3. Besson J (2010) Continuum models of ductile fracture: a review. Int J Damage Mech 19(1):3-52

4. Tasan CC, Hoefnagels JPM, Geers MGD (2012) Identification of the continuum damage parameter: an experimental challenge in modeling damage evolution. Acta Mater 60(8):3581-3589

5. Benzerga AA, Leblond JB (2010) Ductile fracture by void growth to coalescence. Adv Appl Mech 44:169-305

6. Rice JR, Tracey DM (1969) On the ductile enlargement of voids in triaxial stress fields. J Mech Phys Solids 17(3):201-217

7. Weck A, Wilkinson DS, Maire E, Toda H (2008) Visualization by X-ray tomography of void growth and coalescence leading to fracture in model materials. Acta Mater 56(12):2919-2928

8. Tasan CC, Hoefnagels JPM, ten Horn CHLJ, Geers MGD (2009) Experimental analysis of strain path dependent ductile damage mechanics and forming limits. Mech Mater 41(11):1264-1276

9. Tekkaya AE, Ben Khalifa N, Hering O, Meya R, Myslicki S, Walther F (2017) Forming-induced damage and its effects on product properties. CIRP Ann Manuf Technol 66(1):281-284

Publisher's Note Springer Nature remains neutral with regard to jurisdictional claims in published maps and institutional affiliations. 